PAPER

\title{
Involvement of the peripheral nervous system in human prion diseases including dural graft associated Creutzfeldt- Jakob disease
}

\section{Ishida, S Okino, T Kitamoto, M Yamada}

J Neurol Neurosurg Psychiatry 2005;76:325-329. doi: 10.1136/jnnp.2003.035154

See end of article for authors' affiliations

....................

Correspondence to: Dr Masahito Yamada, Department of Neurology and Neurobiology of Aging, Kanazawa University Graduate School of Medical Science, Takara-machi, 13-1, Kanazawa, Ishikawa 9208640, Japan; m-yamada@ med.kanazawa-u.ac.jp

Received

25 December 2003

In revised form

21 May 2004

Accepted 9 June 2004
Objective: To investigate abnormal prion protein ( $\mathrm{PrP})$ deposition in the peripheral nervous system (PNS) in human prion diseases.

Methods: Eight patients with prion diseases were examined: three with sporadic Creutzfeldt-Jakob disease (sCJD), two with dural graft associated CJD (dCJD), one with Gerstmann-Sträussler-Scheinker disease (GSS) with a PrP P102L mutation (GSS102), and two with a P105L mutation (GSS105). An atypical case of SCJD with PrP plaques in the brain presented clinically with peripheral neuropathy, and showed demyelination in $12 \%$ of the teased fibres of the sural nerve. The PNS was investigated by immunohistochemical and western blotting analyses of PrP.

Results: In immunohistochemical studies, granular PrP deposits were detected in some neurones of dorsal root ganglia and a few fibres of peripheral nerves and spinal posterior roots in one sCJD and two dCJD patients, but not in GSS102 or GSS105 patients. The atypical case of sCJD with peripheral neuropathy showed no obvious PrP deposition in the nerves. Western blotting analysis of the PNS from the dCJD patients revealed a small amount of protease $\mathrm{K}$ resistant $\operatorname{PrP}$ in the dorsal root ganglia and peripheral nerves.

Conclusions: Abnormal PrP deposition occurs in the dorsal root ganglia and peripheral nerves in SCJD and dCJD. The PrP deposits in the PNS are not correlated with clinical manifestation of peripheral neuropathy in CJD.
$\mathrm{H}$ uman prion diseases, including Creutzfeldt-Jakob disease (CJD) and Gerstmann-Sträussler-Scheinker disease (GSS), are transmissible and genetic diseases, and involve predominantly the central nervous system (CNS), which shows spongiform changes and abnormal prion protein (PrP) deposits. Some patients with familial and sporadic CJD (SCJD) and GSS show fasciculation, areflexia, glove and stocking type sensory disturbance, and amyotrophy, implying the presence of peripheral neuropathy or motor neuronopathy. ${ }^{1-7}$ Electrophysiological and histological studies supported these clinical signs or subclinical peripheral involvement, and indicated the presence of demyelinating or axonal neuropathy, or loss of anterior horn cells in the spinal cord in human prion diseases. ${ }^{1-4}$ 8-10 However, PrP deposition in the peripheral nervous system (PNS) has not been fully explored in previous studies. ${ }^{1-4}{ }^{8-10}$ In addition, it remains unclear whether peripheral neuropathy is correlated with abnormal PrP deposition in the PNS of patients with CJD and GSS. In the present study, we carried out immunohistochemical and western blotting analyses of PrP in the PNS in SCJD, dural graft associated CJD (dCJD), and GSS, and we discuss the mechanisms of the PNS involvement in human prion diseases.

\section{METHODS \\ Patients}

We studied eight patients with necropsy confirmed prion diseases: three with SCJD, two with dCJD, ${ }^{11}{ }^{12}$ one with GSS with a PrP P102L mutation (GSS102), and two with a PrP P105L mutation (GSS105) (table 1). One patient (patient 3) with SCJD was an atypical case as PrP plaques were detected in the CNS. ${ }^{13}$ Patient 3 presented clinically with dementia, parkinsonism, and pyramidal signs, but no paroxysmal periodic discharges on EEG. ${ }^{13}$ In addition, patient 3 showed myokymia, hyporeflexia in the lower extremities, and decreased nerve conduction velocities without conduction block, indicating the presence of peripheral neuropathy. Of the two patients with dCJD, ${ }^{11}{ }^{12}$ one (patient 5) had atypical clinicopathological features showing PrP plaque formation in the brain, ${ }^{11}$ but had no evidence of peripheral neuropathy. Patient 6 presented classic clinicopathological manifestations of GSS102, including progressive ataxia and dementia, and also showed areflexia in the lower extremities and painful dysaesthesia. ${ }^{5}$ The other patients had no clinical signs of peripheral neuropathy.

None of the patients with SCJD or dCJD had mutations in the PrP gene. With regard to polymorphic sites in the PrP gene, codon 129 was methionine/valine in only two cases of GSS105 (patients 7 and 8), ${ }^{14}$ and the other patients were homozygous for methionine. All patients were homozygous for glutamic acid in codon 219. The patients with SCJD and dCJD showed a type 1 pattern on western blotting analyses of the protease $\mathrm{K}$ resistant $\operatorname{PrP}\left(\operatorname{PrP}^{\mathrm{Sc}}\right)$ in the brain.

\section{Neuropathology}

Spinal cords, dorsal root ganglia, spinal roots at several levels, peripheral nerves (mainly sciatic and femoral), and quadriceps femoris muscles were sampled at necropsy. Sections from the PNS were stained by routine techniques, including Klüver-Barrera and Bodian. The sural nerve of patient 3 was embedded in Epon and stained with toluidine blue; teased fibres were also analysed in the sural nerve.

Abbreviations: CJD, Creutzfeldt-Jakob disease; dCJD, dural graft associated CJD; GSS, Gerstmann-Sträussler-Scheinker disease; PNS, peripheral nervous system; PrP, prion protein; SCJD, sporadic CJD 
Table 1 Summary of the patients

\begin{tabular}{|c|c|c|c|c|c|c|c|c|}
\hline \multirow[b]{2}{*}{ Patient } & \multirow[b]{2}{*}{ Age $(y) /$ sex } & \multirow[b]{2}{*}{ Diagnosis } & \multirow{2}{*}{$\begin{array}{l}\text { Clinical } \\
\text { course }\end{array}$} & \multirow{2}{*}{$\begin{array}{l}\text { Clinical signs of peripheral } \\
\text { neuropathy }\end{array}$} & \multirow{2}{*}{$\begin{array}{l}\text { Patterns of PrP deposits } \\
\text { in CNS }\end{array}$} & \multicolumn{3}{|c|}{ PrP gene analysis } \\
\hline & & & & & & Mutation* & Codon129t & $\overline{\text { Codon219t }}$ \\
\hline 1 & $60 / F$ & sCJD & $4 \mathrm{~m}$ & - & Synaptic & - & $M / M$ & $E / E$ \\
\hline 2 & $70 / M$ & sCJD & $11 \mathrm{~m}$ & - & Synaptic & - & $M / M$ & $\mathrm{E} / \mathrm{E}$ \\
\hline 3 & $76 / F$ & sCJD (atypical) & $11 \mathrm{~m}$ & $\begin{array}{l}\text { Myokymia, hyporeflexia, } \\
\text { decrease in NCV }\end{array}$ & Synaptic >plaque & - & $M / M$ & $E / E$ \\
\hline 4 & $69 / F$ & $d C J D$ & $6 \mathrm{~m}$ & - & Synaptic & - & $M / M$ & $E / E$ \\
\hline 5 & $42 / M$ & dCJD (atypical) & $13 \mathrm{~m}$ & - & Synaptic $>$ plaque & - & $M / M$ & $\mathrm{E} / \mathrm{E}$ \\
\hline 6 & $38 / F$ & GSS102 & $3 y$ & $\begin{array}{l}\text { Dysaesthesia, areflexia in } \\
\text { the legs }\end{array}$ & Plaque > synaptic & P102L & $M / M$ & $E / E$ \\
\hline 7 & $58 / F$ & GSS105 & $8 y$ & - & Plaque > synaptic & P105L & $M / V$ & $E / E$ \\
\hline 8 & $53 / M$ & GSS105 & $11 y$ & - & Plaque $>$ synaptic & P105L & $M / V$ & $\mathrm{E} / \mathrm{E}$ \\
\hline
\end{tabular}

\section{PrP immunohistochemistry}

Immunohistochemical studies to detect PrP deposits were carried out using a mouse monoclonal antibody 3F4 (Senetek, Maryland Heights, Missouri, USA; 1:500) after hydrolytic autoclaving of formalin fixed, paraffin embedded sections $8 \mu \mathrm{m}$ thick. ${ }^{15}$ The same specimens as were examined morphologically were studied by PrP immunohistochemistry. 3F4 is a well characterised antibody that recognises an epitope corresponding to amino acids 109 to 112 of human PrP. ${ }^{16}$ To confirm specificity of the immunohistochemical reaction, an antigen absorption test was carried out simultaneously using a peptide of 106-115 of human PrP. From patient 3 , the femoral nerve was examined for PrP immunohistochemistry. In addition, we used another antiPrP antibody, anti-PrP-95, which recognises residues 95 to 108 of $\mathrm{PrP}^{17}$; the results of PrP immunohistochemistry were shared by both 3F4 and anti-PrP-95.

\section{Western blotting analysis}

The dorsal root ganglia tissues from patients 1, 4, and 5, and the femoral nerve and quadriceps femoris muscle tissues from patients 3 and 5 were homogenised by a procedure including a collagenase digestion step to improve detection of $\mathrm{PrP}^{\mathrm{Sc}},{ }_{18}$ and western blotting analysis of $\mathrm{PrP}^{\mathrm{Sc}}$ was undertaken with $3 \mathrm{~F} 4 .^{19}$

\section{RESULTS}

\section{Neuropathology}

No neuronal loss or histological abnormalities were observed in any PNS tissues from patients 1,2 , or $4-8$, or in the femoral nerve from patient 3 . The sural nerve from patient 3 showed no apparent loss of myelinated fibres, but demyelination was seen in $12 \%$ of all the teased fibres.

\section{PrP immunohistochemistry}

The results of PrP immunohistochemistry are shown in table 2 .

\section{Spinal cord}

Granular PrP deposits were observed in the posterior horns of the spinal cords, particularly in the substantia gelantinosa, of all patients with SCJD and dCJD (patients 1 to 5 ), and of one with GSS102 (patient 6) (fig 1, panels A and B). There were no granular deposits of PrP in the spinal cords of two subjects with GSS105 (patients 7 and 8). Only patient 6 with GSS102 and patient 8 with GSS105 showed a few PrP positive plaques in the spinal posterior horns (fig 1), as reported previously. ${ }^{5}$

\section{Dorsal root ganglia}

Granular PrP deposits were detected inside and on the cell surfaces of some dorsal root ganglia neurones in one of two sCJD patients examined (patient 2), and in both of the dCJD cases (patients 4 and 5) (fig 2). Patient 1 with SCJD, patient 6 with GSS102, and patients 7 and 8 with GSS105 showed no PrP deposits in the dorsal root ganglia.

\section{Spinal roots}

Patients 2, 4, and 5-who had PrP deposits in dorsal root ganglia-showed PrP immunoreactivity in some of the spinal roots (fig 3A). No PrP deposits were detected in the spinal roots in the patients who showed no PrP deposits in the dorsal root ganglia.

\section{Peripheral nerves}

PrP was detected in the peripheral nerves, particularly in the axons of some nerves, from patients 4 and 5 (fig 3B). Patients 4 and 5 also showed PrP deposition in the dorsal root ganglia

Table 2 Results of immunohistochemical analysis for prion protein deposition

\begin{tabular}{|c|c|c|c|c|c|c|c|c|}
\hline \multirow[b]{2}{*}{ Patient } & \multirow[b]{2}{*}{$\begin{array}{l}\text { Age }(y) / \\
\text { sex }\end{array}$} & \multirow[b]{2}{*}{ Diagnosis } & \multicolumn{2}{|c|}{ Spinal cord } & \multirow[b]{2}{*}{$\begin{array}{l}\text { Dorsal root } \\
\text { ganglia }\end{array}$} & \multirow[b]{2}{*}{ Roots } & \multirow[b]{2}{*}{ Nerves } & \multirow[b]{2}{*}{ Muscles } \\
\hline & & & $\begin{array}{l}\text { Synaptic } \\
\text { type }\end{array}$ & $\begin{array}{l}\text { Plaque } \\
\text { type }\end{array}$ & & & & \\
\hline 1 & $60 / F$ & SCJD & $+(\mathrm{PH})$ & - & - & - & - & - \\
\hline 2 & $70 / M$ & SCJD & $+(\mathrm{PH})$ & - & + & + & NE & NE \\
\hline 3 & $76 / F$ & SCJD & $+(\mathrm{PH})$ & - & NE & - & - & - \\
\hline 4 & $69 / F$ & dCJD & $+(\mathrm{PH})$ & - & + & + & + & NE \\
\hline 5 & $42 / M$ & $\mathrm{dCJD}$ & $+(\mathrm{PH})$ & - & + & + & + & - \\
\hline 6 & $38 / F$ & GSS102 & $+(\mathrm{PH})$ & + & - & - & - & NE \\
\hline 7 & $58 / F$ & GSS105 & - & - & - & - & - & NE \\
\hline 8 & $53 / M$ & GSS105 & - & + & - & - & - & - \\
\hline
\end{tabular}



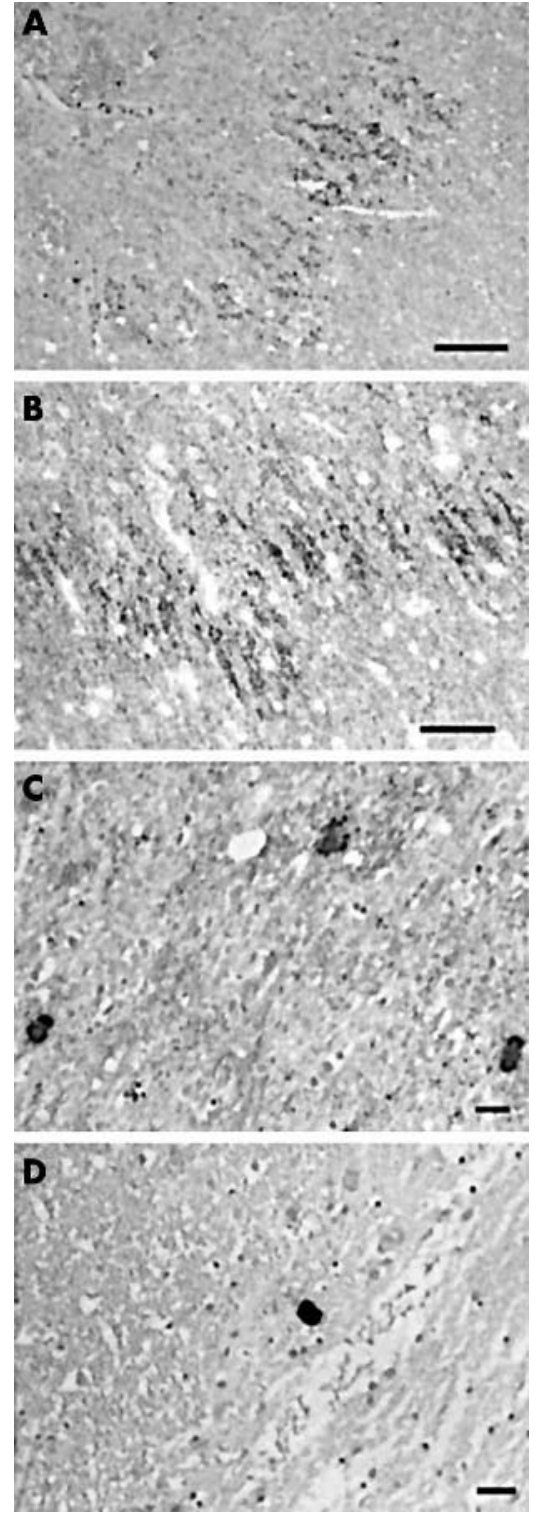

Figure 1 Immunohistochemical findings in the substantia gelantinosa in spinal posterior horns using an anti-prion protein $(\mathrm{PrP})$ monoclonal antibody, 3F4. Granular PrP deposits were detected in patient 2 with sporadic Creutzfeldt-Jakob disease (CJD) (panel A), and patient 4 with dural graft associated CJD (panel B). (C) Patient 6 with GerstmannSträussler-Scheinker disease (GSS) having a P102L mutation showed both granular PrP deposits and a few PrP plaques. (D) Patient 8 with GSS having a P105L mutation showed only plaque type PrP deposits. Scale bars in panels $A$ and $B, 100 \mu \mathrm{m}$. Scale bars in panels $C$ and D, $20 \mu \mathrm{m}$.

and the spinal roots. Patients 3 and 6 with clinical signs of peripheral neuropathy showed no PrP deposits in the peripheral nerves.

\section{Muscles}

None of the muscles examined from patients $1,3,5$, and 8 showed PrP immunoreactivity.

\section{Western blotting analysis}

$\mathrm{PrP}^{\mathrm{SC}}$ with a type 1 pattern was detected on western blotting analysis of the dorsal root ganglia, and was seen at a very low level in the peripheral nerve from patient 5 with dCJD, but not in the others (fig 4). The femoral nerve and quadriceps femoris muscle from patient 3 showed no $\operatorname{PrP}^{\mathrm{SC}}$ positive bands (data not shown).
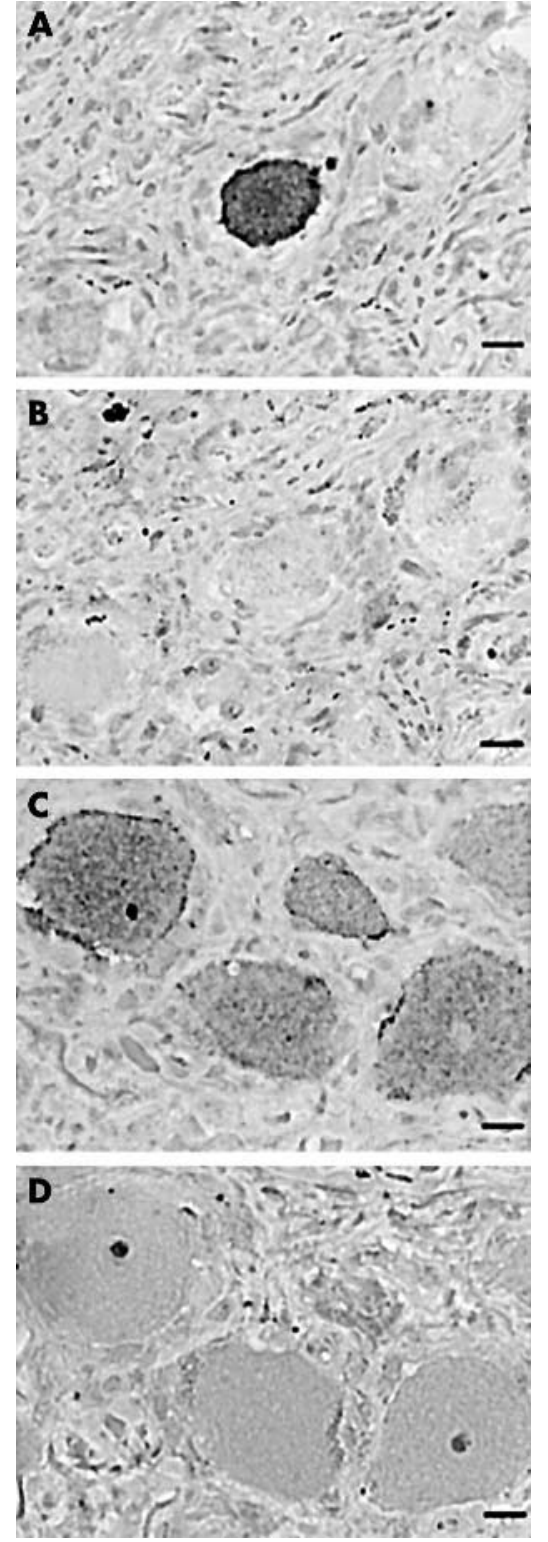

Figure 2 Immunohistochemical findings of dorsal root ganglia using an anti-prion protein (PrP) monoclonal antibody, 3F4, in patient 2 with sporadic Creutzfeldt-Jakob disease (CJD) (panels $A$ and $B$ ), and in patient 5 with dural graft associated CJD (panels $C$ and D). PrP deposits were detected in some dorsal root ganglia cells from patient 2 (A) and from patient 5 (C). PrP immunoreactivity was abolished by preabsorption of the antibody with a peptide corresponding to residues 106 to 115 of human $\operatorname{PrP}(B$ and D). Scale bars, $100 \mu \mathrm{m}$.

\section{DISCUSSION}

Our immunohistochemical studies indicated that PrP deposition occurred in the dorsal root ganglia and peripheral nerves in SCJD and dCJD. Further, western blotting analysis revealed $\mathrm{PrP}^{\mathrm{Sc}}$ in the dorsal root ganglia and the peripheral nerves in dCJD.

Extensive accumulation of $\mathrm{PrP}^{\mathrm{Sc}}$ in the dorsal root ganglia and autonomic ganglia has been reported in variant CJD (vCJD) ${ }^{20-22}$ In non-iatrogenic CJD, some immunohistochemical studies have revealed PrP deposits in the PNS. ${ }^{21} 2324 \mathrm{~A}$ patient with possibly familial CJD showed PrP immunoreactivity in the posterior root nerve fibres in an adaxonal location, although western blotting analysis was not undertaken in this case. ${ }^{23}$ In a valine homozygous type- 1 (VVI) case of sporadic CJD, linear PrP deposits were detected in the 
posterior roots and along nerve fibres of both myelinated and unmyelinated fibres in the sciatic and peroneal nerves. ${ }^{24}$ However, western blotting analysis showed the absence of $\mathrm{PrP}^{\mathrm{SC}}$ in this VVI case of sCJD. ${ }^{24}$ With regard to SCJD patients except for VVl cases, three of six patients showed PrP deposits in the dorsal root ganglia, while no PrP deposition has been found in the peripheral nerves of any patients. ${ }^{21}$ Our results support those of previous reports indicating the presence of PrP deposition in the dorsal root ganglia and

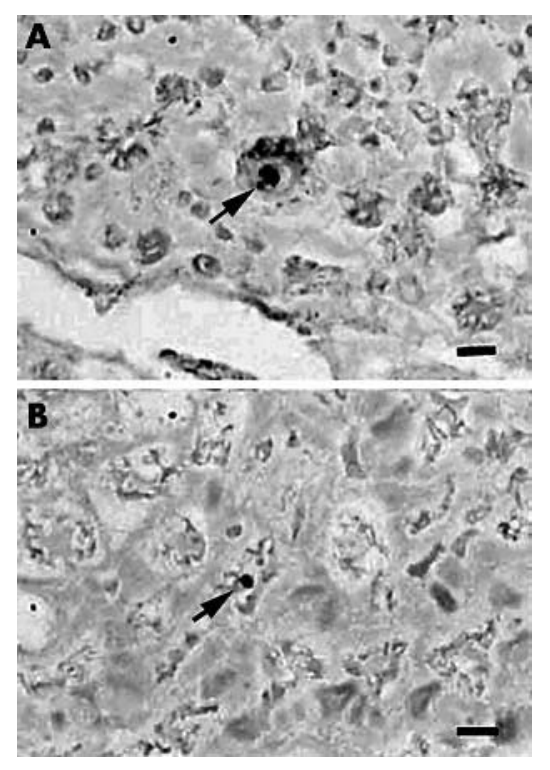

Figure 3 Immunohistochemical findings in a posterior root from patient 2 with sporadic Creutzfeldt-Jakob disease (CJD) (A) and a peripheral nerve from patient 5 (B) with dural graft associated CJD using an antiprion protein (PrP) monoclonal antibody, 3F4. Some nerve fibres, particularly in the axons surrounded by myelin sheaths, showed PrP immunoreactivity (arrows). Scale bars, $1 \mu \mathrm{m}$.

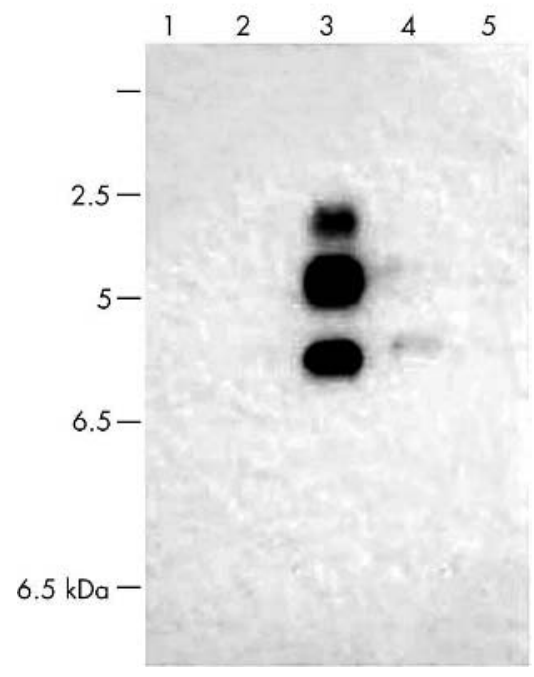

Figure 4 Western blotting analysis using $3 \mathrm{~F} 4$ of protease $\mathrm{K}$ resistant fractions from dorsal root ganglia (DRG) of patient 1 (lane 1), DRG of patient 4 (lane 2), and DRG (lane 3), peripheral nerve (lane 4), and skeletal muscle (lane 5) of patient 5. No bands were detected in the DRG of patient 1 with sporadic Creutzfeldt-Jakob disease (CJD), or patient 4 with dural graft associated CJD (dCJD) (lanes 1 and 2). In patient 5 with dCJD, the DRG tissue showed significant immunoreactivity with 3F4 (lane 3), and the peripheral nerve showed faint immunoreactivity (lane 4). The skeletal muscle from patient 5 showed no immunoreactivity (lane 5). peripheral nerves in sCJD. ${ }^{21}{ }^{24}$ However, patient 2 in the present study is the first case of methionine homozygous type-1 (MMI) SCJD showing PrP deposits in the PNS, because the previous report did not refer to the PrP phenotypes of the SCJD patients. ${ }^{21}$ There was no significant correlation between the duration of the clinical course and the presence of PrP deposits.

Our results indicated a novel finding regarding the presence of $\mathrm{PrP}^{\mathrm{SC}}$ in the dorsal root ganglia and peripheral nerves in dCJD. For iatrogenic CJD, PrP deposits have been reported only in the spinal cords of growth hormone related cases and of a patient with dCJD, but not in the PNS. ${ }^{25} 26$ Another earlier study showed no $\operatorname{PrP}^{\mathrm{Sc}}$ deposits in the dorsal root ganglia or the peripheral nerves of a patient with growth hormone related CJD. ${ }^{21}$

With regard to GSS (GSS102 and GSS105), no PrP deposits were found in the dorsal root ganglia or nerves in the present study, although the posterior horns of the spinal cord were affected by PrP. ${ }^{514}$ Our results suggest that there are differences in PrP accumulation in the PNS between CJD and GSS. In skeletal muscles, we found no PrP immunoreactivity or $\mathrm{PrP}^{\mathrm{SC}}$ accumulation by western blotting analysis. These results differ from those of a recent report, which showed the presence of $\mathrm{PrP}^{\mathrm{Sc}}$ in skeletal muscle from eight of 32 patients with sCJD. ${ }^{27}$ However, our procedure of $\mathrm{PrP}^{\mathrm{Sc}}$ analysis with collagenase digestion of samples ${ }^{18}$ was different from the one in this previous study which used precipitation with phosphotungstic acid, ${ }^{27}$ and our negative results were limited to only four patients - two with SCJD, one with dCJD, and one with GSS105.

PrP deposition is observed in the PNS in natural ${ }^{28} 29$ and experimental scrapie, ${ }^{30-32}$ bovine spongiform encephalitis, ${ }^{33}$ and variant CJD (vCJD). ${ }^{20-22}$ These previous studies suggest that the PNS can be a neural pathway to the CNS following oral exposure to a prion agent. The strong PrP immunopositivity (consisting of $\mathrm{PrP}^{\mathrm{SC}}$ ) of the autonomic and dorsal root ganglia in vCJD may also support a peripheral pathogenesis. ${ }^{20-22}$ However, our results showed $\operatorname{PrP}^{\mathrm{SC}}$ deposits in the PNS in dCJD. dCJD might be caused by direct attachment of contaminated cadaveric dura mater to the brain surface, because some patients with dCJD are associated with initial clinical manifestations ${ }^{12}$ or abnormalities on magnetic resonance imaging in the dura mater grafted sites (Satoh $\mathrm{T}$, personal communication). These findings suggest that $\operatorname{PrP}$ deposits in the PNS do not necessarily provide evidence of peripheral pathogenesis in human prion diseases.

Our results also imply that the PrP deposits in the PNS are not necessarily correlated with clinical presentations of peripheral neuropathy in CJD. In the present study, two patients-patient 3 with SCJD and patient 6 with GSS102presented with clinical manifestations suggesting involvement of the PNS. Patient 6 had dysaesthesia and areflexia in the lower extremities, which could have reflected involvement of the posterior horn with PrP deposits. Patient 3 showed myokymia, hyporeflexia in the lower extremities, decreased conduction velocities in nerve conduction studies, and some demyelination in the teased fibres. Nerve conduction studies in some CJD patients showed decreases in conduction velocity, ${ }^{1-368}$ multifocal conduction block, ${ }^{1}$ low occurrence of F waves, ${ }^{7}$ abnormalities of sensory evoked potentials, ${ }^{7}$ diminished compound muscle action potential and sensory nerve action potential, and prolonged distal latency. ${ }^{2}$ Fibrillation, fasciculation, and polyphasic motor unit potentials have also been reported in electromyograms in patients with CJD. ${ }^{18}$ The results of histological studies have supported these electrophysiological abnormalities in only a few patients. ${ }^{138}$ In addition, no PrP immunoreactivity was detected in peripheral nerves from CJD patients with histologically confirmed demyelination, which was 
consistent with the findings in patient 3 in the present study. ${ }^{139}$ It is unlikely that peripheral neuropathy reported in some CJD patients ${ }^{1-10}$ is an incidental finding unrelated to CJD. We speculate that peripheral neuropathy in CJD may not be caused directly by abnormal PrP accumulation in the PNS. Transgenic mice overexpressing wild-type PrP showed polyneuropathy, although PrP deposits were not examined in the PNS. ${ }^{34}$ It is possible that abnormal metabolism of PrP in the PNS without apparent accumulation of $\operatorname{PrP}^{\mathrm{Sc}}$ may be responsible for peripheral neuropathy in CJD, although the precise mechanism remains to be determined. Further clinical and experimental studies are necessary to elucidate the pathomechanism underlying peripheral neuropathy in CJD.

\section{Authors' affiliations}

C Ishida, S Okino, M Yamada, Department of Neurology and Neurobiology of Aging, Kanazawa University Graduate School of Medical Science, Kanazawa, Japan

T Kitamoto, Department of Neurological Science, Tohoku University Graduate School of Medicine, Sendai, Japan

Competing interests: none declared

\section{REFERENCES}

1 Neufeld MY, Josiphov J, Korczyn AD. Demyelinating peripheral neuropathy in Creutzfeldt-Jakob disease. Muscle Nerve 1992;15:1234-9.

2 Niewiadomska M, Kulczycki J, Wochnik-Dyjas D, et al. Impairment of the peripheral nervous system in Creutzfeldt-Jakob disease. Arch Neurol 2002;59:1430-6.

3 Antoine JC, Laplanche JL, Mosnier JF, et al. Demyelinating peripheral neuropathy with Creutzfeldt-Jakob disease and mutation at codon 200 of the prion protein gene. Neurology 1996;46:1123-7.

4 Worrall BB, Rowland LP, Chin SM, et al. Amyotrophy in prion diseases. Arch Neurol 2000:57:33-8.

5 Yamada $M$, Tomimitsu $H$, Yokota $Y$, et al. Involvement of the spinal posterior horn in Gerstmann-Sträussler-Scheinker disease (PrP P102L). Neurology 1999;52:260-5

6 Esiri MM, Gordon WI, Collinge J, et al. Peripheral neuropathy in CreutzfeldtJakob disease. Neurology 1997;47:784.

7 Takase K, Furuya H, Murai H, et al. A case of Gerstmann-Sträussler-Scheinker syndrome (GSS) with late onset-A haplotype analysis of Glu219lys polymorphism in PrP gene-. Rinsho Shinkei 2001;41:318-21 [In Japanese with English abstract].

8 Sadeh M, Chagnac Y, Goldhammer Y. Creutzfeldt-Jakob disease associated with peripheral neuropathy. Isr J Med Sci 1990;26:220-2.

9 Vallet J, Dumas M, Corvisier N, et al. Familial Creutzfeldt-Jakob disease with extensive degeneration of white matter: ultrastructure of peripheral nerve. J Neurol Sci 1983;61:261-75.

10 Chapman J, Brown P, Goldfarb LG, et al. Clinical heterogeneity and unusual presentations of Creutzfeldt-Jakob disease in Jewish patients with the PRNP codon 200 mutation. J Neurol Neurosurg Psychiatry 1993;56:1 109-12.

11 Kimura K, Nonaka A, Tashiro H, et al. Atypical form of dural graft associated Creutzfeldt-Jakob disease: report of a postmortem case with review of the literature. J Neurol Neurosurg Psychiatry 2001;70:696-9.
12 Nishida Y, Yamada M, Hara K, et al. Creutzfeldt-Jakob disease after Jannetta's operation with cadaveric dura mater graft: initial manifestations related to the grafted site. J Neurol 2002;249:480-3.

13 Ishida C, Kakishima A, Okino S, et al. Sporadic Creutzfeldt-Jakob disease with MM1-type prion protein and plaques. Neurology 2003:60:514-17.

14 Yamada M, Itoh Y, Inaba A, et al. An inherited prion disease with a PrP P105L mutation: clinicopathologic and PrP heterogeneity. Neurology 1999;53:181-8.

15 Yamada $M$, Satoh S, Sodeyama $N$, et al. Spastic paraparesis and mutations in the prion protein gene. J Neurol Sci 1995;134:215-16.

16 Kitamoto T, Shin R-W, Doh-ura K, et al. Abnormal isoform of prion proteins accumulates in the synaptic structures of the central nervous system in patients with Creutzfeldt-Jakob disease. Am J Pathol 1992;140:1285-94.

17 Yamada M, Itoh Y, Suematsu N, et al. Panencephalopathic type of Creutzfeldt-Jakob disease associated with cadaveric dura mater graft. J Neurol Neurosurg Psychiatry 1997;63:524-7.

18 Grathwohl KU, Horiuchi M, Ishiguro N, et al. Improvement of PrPSc-detection in mouse spleen early at the preclinical stage of scrapie with collagenasecompleted tissue homogenization and Sarkosyl- $\mathrm{NaCl}$ extraction of $\mathrm{PrPSc}$. Arch Virol 1996;141:1863-74.

19 Simizu S, Hoshi K, Muramoto T, et al. Creutzfeldt-Jakob disease with floridtype plaques after cadaveric dura mater grafting. Arch Neurol 1999;56:357-62

20 Ironside JW. Pathology of variant Creutzfeldt-Jakob disease. Arch Virol 2000;16(suppl): 143-51

21 Head MW, Ritchie D, Smith N, et al. Peripheral tissue involvement in sporadic, iatrogenic, and variant Creutzfeldt-Jakob disease: an immunohistochemical, quantitative, and biochemical study. Am J Pathol 2004;164:143-53.

22 Haik S, Faucheux BA, Sazdovitch V, et al. The sympathetic nervous system is involved in variant Creutzfeldt-Jakob disease. Nat Med 2003;9:1121-3.

23 Hainfellner JA, Budka H. Disease associated prion protein may deposit in the peripheral nervous system in human transmissible spongiform encephalopathies. Acta Neuropathol 1999;98:458-60.

24 Favereaux A, Quadrio I, Perret-Liaudet A, et al. Prion protein accumulation involving the peripheral nervous system in a sporadic case of CreutzfeldtJakob disease. Neuropathol Appl Neurobiol 2003;29:602-5.

25 Goodbrand IA, Ironside JW, Nicolson D, et al. Prion protein accumulation in the spinal cords of patients with sporadic and growth hormone associated Creutzfeldt-Jakob disease. Neurosci Lett 1995;183:127-30.

26 Radbaver C, Hainfellner JA, Gaudernak T, et al. Creutzfeldt-Jakob disease in a dura transplant recipient: first observation in Austria. Wien Klin Wochenschr 1998; 110:496-500 [In German with English abstract].

27 Glatzel M, Abela E, Maissen M, et al. Extraneural pathologic prion protein in sporadic Creutzfeldt-Jakob disease. N Engl J Med 2003;349:1812-20.

28 Groschup MH, Weiland F, Straub OC, et al. Detection of scrapie agent in the peripheral nervous system of a diseased sheep. Neurobiol Dis 1996;3:191-5.

29 Hadlow JW, Race RE, Kennedy RC, et al. Natural infection of the sheep with scrapie virus. In: Prusiner SB, Hadlow WJ, eds. Slow transmissible diseases of the nervous system, vol 2. New York: Academic press, 1979:331-56.

30 Groschup MH, Beekes M, McBride PA, et al. Deposition of disease-associated prion protein involves the peripheral nervous system in experimental scrapie. Acta Neuropathol 1999:98:453-7.

31 McBride PA, Beekes M. Pathological PrP is abundant in sympathetic and sensory ganglia of hamsters fed with scrapie. Neurosci Lett 1999;265:135-8.

32 Beekes M, McBraode PA, Baldauf E. Cerebral targeting indicates vagal spread of infection in hamsters fed with scrapie. J Gen Virol 1998;79:601-7.

33 Wells GA, Hawkins SA, Green RB, et al. Preliminary observations on the pathogenesis of experimental BSE: an update. Vet Rec 1998;142:103-6.

34 Westaway D, DeArmond SJ, Cayetano-Canlas J, et al. Degeneration of skeletal muscle, peripheral nerves, and the central nervous system in transgenic mice overexpressing wild-type prion proteins. Cell 1994;76: 117-29. 